\title{
Analyzing Kansei from Facial Expressions by CSRBF Mapping
}

\author{
Luis Diago $^{1,2}$, Julian Romero ${ }^{1,2}$, Junichi Shinoda ${ }^{1}$ and Ichiro Hagiwara ${ }^{2}$ \\ 1. Interlocus Inc, TokyoTech YVP W305, Yokohama 226-8510, Japan \\ 2. Meiji Institute for Advanced Studies of Mathematical Sciences, Meiji University, Tokyo 164-8525, Japan
}

\begin{abstract}
This paper describes an application where a new KAE (Kansei/Affective Engineering) system was applied to define the properties of the facial images perceived as Iyashi. Iyashi is a Japanese word used to describe a peculiar phenomenon that is mentally soothing, but is yet to be clearly defined. Instead of analyzing facial expressions of an individual to determine his emotional state, the proposed system introduces a FQHNN (fuzzy-quantized holographic neural network) to find the rules involved in the Kansei evaluation provided by the subjects about the limited dataset of 20 facial images. In order to validate and gain a clear insight into the rules involved in the Kansei evaluation process, Procrustes analysis and CSRBFs (compactly-supported radial basis functions) are combined to generate new facial images. Procrustes analysis is used to find the minimal dissimilarity measure between two facial images with opposite classification (i.e., Iyashi and Non-Iyashi). CSRBFs are proposed for tuning of 17 facial parameters and mapping between facial images within opposite classes. The experiments with two subjects demonstrate that if only two from the five most important parameters of the face are changed, then the Kansei evaluation can change to the opposite class. This paper shows that a continuous and efficient tuning of the design space can be achieved by introducing CSRBF mapping into the new KAE system.
\end{abstract}

Key words: Kansei evaluation, Iyashi expressions, neuro-fuzzy classifiers, radial basis functions.

\section{Introduction}

In an attempt to understand what motivates people to buy consumer products, product manufacturers often rely on questionnaires and polls to investigate the consumers' preferences and why they purchase or avoid certain items [1]. The major problem with this approach is that, as shown by a variety of studies e.g., Ref. [1], consumer attitudes and behaviors are frequently formed and influenced by non-conscious emotional and cognitive processing [2], which is not subject to detection via traditional survey or interview methods. Here, KAE (Kansei/Affective Engineering) systems [3] assume that product-related stimuli (e.g., paintings or photographs) are shown as questionnaires to the consumers and at the same time a machine automatically recognizes and analyzes interactive signals while consumers evaluate what they felt about products in photographs. Then, using simple (e.g.,

Corresponding author: Luis Diago, Ph.D., research fields: kansei engineering and robotics. E-mail: ldiago@i-locus.com. "yes/no") answers to evaluate Kansei words, a comprehensive analysis of those results could play an important role during the analysis of consumer state of mind (cognitive and emotional reaction, both conscious and un-conscious) at the time of review, selection or use of a product or product-related stimuli.

Psychological researchers use diverse methods to investigate emotions [4-6]. These procedures range from imagery inductions to film clips and static pictures. One of the most widely used stimulus sets is the IAPS (international affective picture system) [5], a set of static images based on a dimensional model of emotion [7]. The image set contains various pictures depicting mutilations, snakes, insects, attack scenes, accidents, contamination, illness, loss, pollution, puppies, babies, and landscape scenes, among others. However, while many samples are desirable for estimating the response of a person more accurately (e.g., how much the person likes a product), in a real 
world situation, only a small number of samples needs to be obtained because of the efforts required for the persons to provide their responses from many samples. Hence, in this paper as in our previous works [8], we use a small dataset of portraits to teach the machine to classify the facial images in the same way that people perceive them.

Facial expressions are our primary means of communicating emotion [9], and that is why the majority of efforts in affective computing concern automatic analysis of facial displays [11]. Instead of analyzing facial expressions of an individual to determine their emotional state, using a database of portraits, Diago, et al [8] showed that machines are also capable of perceiving faces as they relate to the social impressions they make on people. A new method for the quantification of qualitative judgments and evaluations of facial expressions was proposed in order to gain a clear insight into the reasoning made by nonlinear prediction models, such as HNN (holographic neural networks).

In previous works [14], the term "Iyashi ${ }^{1}$ expressions" was defined to study facial expressions related with the peculiar phenomenon of Iyashi. In Japan, Iyashi is a popular buzzword today, referring to anything that is physically or mentally soothing [14]. Iyashi goods-books, music, pictures, incense and aromas, bath salts, and plants - abound, offer to heal the physical and psychological stress of the workplace and of daily life in general. In society, the expression Iyashi-kei is frequently used to describe laypersons that simply help people to relax. However, the word Iyashi (like other such as pleasure, well-being or satisfaction) does not have a consensus among people. Therefore, a KAE system was developed to explain human decision-making by extracting fuzzy rules from the computational models. In this paper, Procrustes analysis and CSRBFs (compactly-supported radial basis functions) are

\footnotetext{
${ }^{1}$ We use the word Iyashi as the stimulus given to a person that changes its internal condition to a better physical or emotional state (see Ref. [14] for details).
}

combined to validate the extracted rules making a continuous and efficient tuning of the design space.

The paper is organized as follows: Section 2 gives a review of the related KAE systems; Section 3 presents the proposed FQHNN (fuzzy-quantized holographic neural network); Section 4 introduces the combination of Procrustes analysis with CSRBFs; Section 5 shows the experimental results with two subjects; finally, Section 6 presents the conclusions and future works.

\section{Related KAE Systems}

Various KAE Systems have been developed to automatically support the design process [3]. In this Section, we do not intend to do a thorough analysis on existing KAE systems but mention some of the methods or systems including similar applications. For example, Benitez, et al [16] developed a KAE system for Gigakuman Character Design. The system generates different facial expressions of Gigakuman in order to represent an abstract concept. Ohira, et al [17] also use a rough set approach to extract painting composition rules and synthesize drawing and painting from the identification of salient features within images to produce an abstract composition. A more sophisticated system was proposed by Ando and Hagiwara [18] to create 3D characters using interactive genetic algorithm and a rule extraction method based on the comparison between attributes of user's evaluated characters. Recently, the generation of Kansei rules trends to include parameters from physiological measurements [19], but we will not cover this topic here.

Fuzzy logics, neural networks, genetic algorithm, rough-set analysis, and partial least square analysis are used within KAE systems to analyze Kansei [3]. A neuro-fuzzy approach is used in the proposed KAE system [8]. Twenty images represented by seventeen parameters were evaluated by one hundred and fourteen subjects between 15 and 70 years old (102 Japanese and 12 non-Japanese, 47 females and 67 males) and were used to train different neuro-fuzzy 
classifiers. The participants rated each stimulus on the scale ' 0 '- NO, ' 1 '-DON'T KNOW, '2'-YES to express whether or not they feel an Iyashi-stimulus. Fig. 1 shows 63 feature points used to compute the set of parameters describing 20 portrait images.

\section{FQHNN}

Fig. 2 shows the architecture of the proposed FQHNN. The HNN module (upper part) is combined with the FQ module (lower part) to increase HNN interpretability. HNN module is proposed to select the most important parameters of the face from the complex values stored in the holographic memory $(\mathbf{H})$. FQ module is proposed to build the MFs (membership functions) describing facial parameters $\left(\mu_{11}, \ldots, \mu_{k l i}\right)$ and the groups of subject evaluations $\left(\mu_{G 1}, \ldots, \mu_{G m}\right)$ without solving a generalize eigenvalue problem (Ac $\left.=\eta^{2} \mathbf{B c}\right)$. Later, the fuzzy reasoning is used to provide transparency to HNN models extracting linguistic rules [8].

An initial knowledge base of 35 rules was created from the trained FQHNN representing the evaluations of $N=87$ subjects reported in Ref. [8]. Table 1 shows eleven parameters $\left(x_{1}, x_{3}, x_{5}, x_{6}, x_{8}, x_{10}, x_{11}, x_{13}, x_{14}, x_{15}\right.$, $\left.x_{17}\right)$ used to explain the meaning of subjects evaluations from the set of 21 rules extracted from $N$ $=41$ subjects using a single parameter in the antecedent. The first row displays the category ( $\mathrm{S}=$ small, $\mathrm{M}=$ medium, $\mathrm{L}=$ large) of each parameter in the rules. The second row shows the number of classes involved in each parameter. The class is specified between parentheses in case of a single class (' 0 '-NO, ' 1 '-DON'T KNOW, ' 2 '-YES). The third and fourth rows show the number of rules and the number of subjects in which each parameter defined the evaluation.

Table 2 shows fuzzy rules corresponding to the five parameters $\left(x_{5}, x_{6}, x_{11}, x_{13}, x_{17}\right)$ influencing the evaluation of many subjects $(\sim 80 \%)$. Each row represents a rule. Columns 3 and 5 represent the antecedents and consequences of each rule respectively.
Column 6 shows the number of subjects in which the rule defined the evaluation. According to the number of subjects (11), $x_{5}$ is the most influential parameter. However, the rules are contradictory. The same applies to $x_{6}$ and $x_{17}$. In over $80 \%$ of cases $\mathrm{S}$ or $\mathrm{M}$, values of the parameters $x_{11}$ and $x_{13}$ do not produce

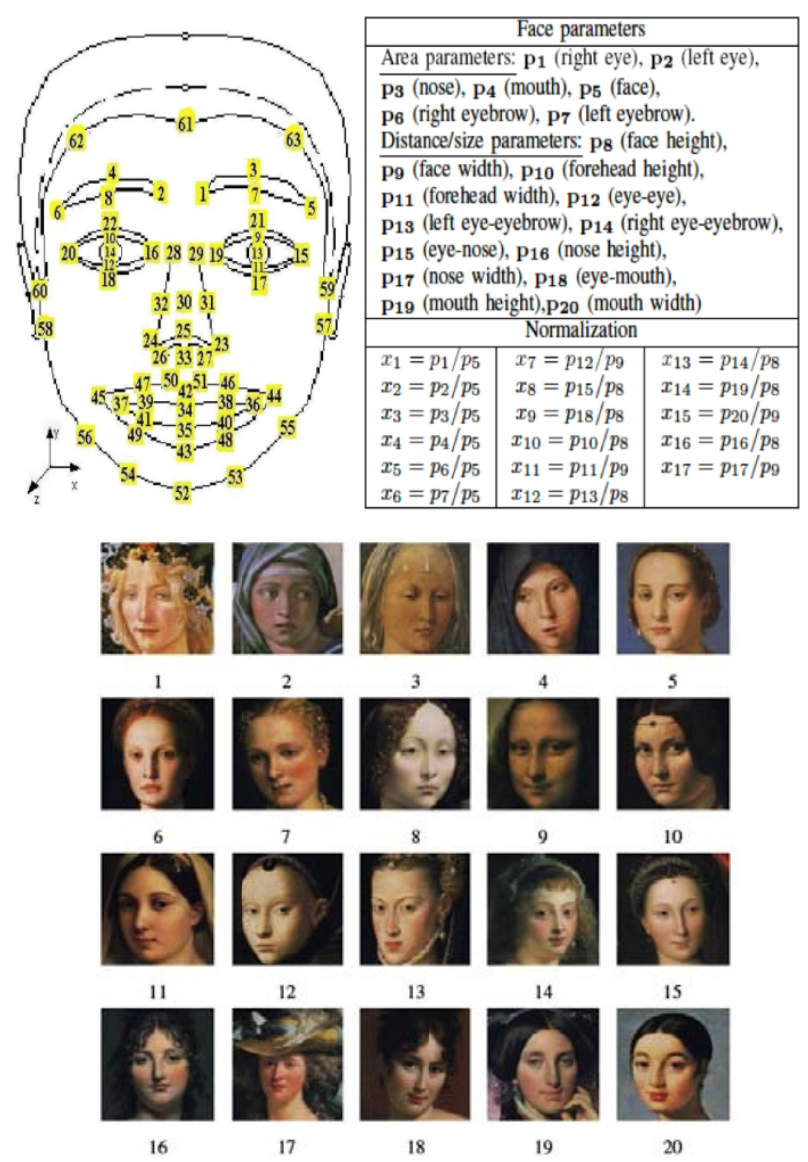

Fig. 1 Feature points, face parameters and portraits.

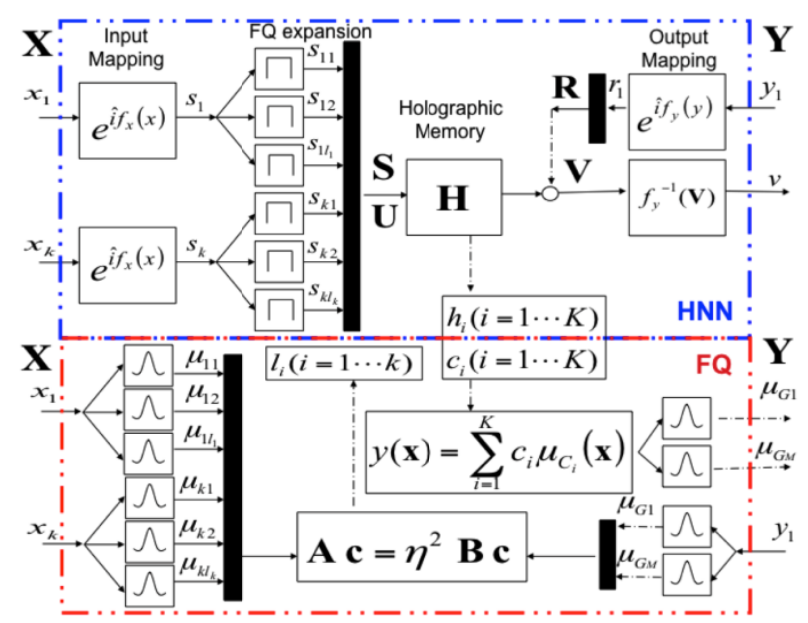

Fig. 2 Architecture of the proposed FQHNN. 
Table 1 Eleven parameters used to explain the meaning of subjects evaluations of Iyashi-expressions.

\begin{tabular}{llllllllllll}
\hline & $x_{1}$ & $x_{3}$ & $x_{5}$ & $x_{6}$ & $x_{8}$ & $x_{10}$ & $x_{11}$ & $x_{13}$ & $x_{14}$ & $x_{15}$ & $x_{17}$ \\
\hline Categories & $\mathrm{M}$ & $\mathrm{S}$ & $\mathrm{S}, \mathrm{M}$ & $\mathrm{M}, \mathrm{L}$ & $\mathrm{M}$ & $\mathrm{M}$ & $\mathrm{S}, \mathrm{M}$ & $\mathrm{M}$ & $\mathrm{S}$ & $\mathrm{M}$ & $\mathrm{S}, \mathrm{M}$ \\
No. of. Classes & $1(1)$ & $1(0)$ & 3 & 2 & $1(1)$ & $1(0)$ & $1(0)$ & 2 & $1(0)$ & $1(2)$ & 2 \\
No. of. Rules & 1 & 1 & 5 & 3 & 1 & 1 & 2 & 2 & 1 & 1 & 3 \\
No. of. Subjects & 1 & 1 & 11 & 8 & 1 & 2 & 4 & 7 & 1 & 2 & 3 \\
\hline
\end{tabular}

Table 2 Fuzzy rules to classify Iyashi-expressions.

\begin{tabular}{|c|c|c|c|c|c|c|}
\hline \multicolumn{2}{|c|}{ No. } & \multicolumn{2}{|l|}{ Parameter } & \multirow{2}{*}{$\begin{array}{l}\text { Class (Iyashi) } \\
\text { NO }\end{array}$} & \multicolumn{2}{|c|}{ No. of Subjects } \\
\hline 1 & \multirow{5}{*}{ If } & $x_{5}$ is $\mathrm{S}$ & \multirow{5}{*}{ Then } & & 4 & \multirow{5}{*}{11} \\
\hline 2 & & $x_{5}$ is $\mathrm{M}$ & & $\mathrm{NO}$ & 1 & \\
\hline 3 & & $x_{5}$ is $\mathrm{M}$ & & DON'T KNOW & 2 & \\
\hline 4 & & $x_{5}$ is $\mathrm{S}$ & & YES & 2 & \\
\hline 5 & & $x_{5}$ is $\mathrm{M}$ & & YES & 2 & \\
\hline 6 & \multirow{3}{*}{ If } & $x_{6}$ is $\mathrm{M}$ & \multirow{3}{*}{ Then } & NO & 5 & \multirow{3}{*}{8} \\
\hline 7 & & $x_{6}$ is $\mathrm{M}$ & & YES & 1 & \\
\hline 8 & & $x_{6}$ is $\mathrm{L}$ & & YES & 2 & \\
\hline 9 & \multirow{2}{*}{ If } & $x_{11}$ is $\mathrm{S}$ & \multirow{2}{*}{ Then } & $\mathrm{NO}$ & 2 & \multirow{2}{*}{4} \\
\hline 10 & & $x_{11}$ is $\mathrm{M}$ & & NO & 2 & \\
\hline 11 & \multirow{2}{*}{ If } & $x_{13}$ is $\mathrm{M}$ & \multirow{2}{*}{ Then } & NO & 5 & \multirow{2}{*}{7} \\
\hline 12 & & $x_{13}$ is $\mathrm{M}$ & & DON'T KNOW & 2 & \\
\hline 13 & \multirow{3}{*}{ If } & $x_{17}$ is $\mathrm{S}$ & \multirow{3}{*}{ Then } & NO & 1 & \multirow{3}{*}{3} \\
\hline 14 & & $x_{17}$ is $\mathrm{S}$ & & YES & 1 & \\
\hline 15 & & $x_{17}$ is $\mathrm{M}$ & & YES & 1 & \\
\hline
\end{tabular}

Iyashi, i.e., Class (Iyashi) = NO. In the next section, changing the parameters $x_{6}$ and $x_{17}$, new expressions are generated in order to validate the extracted rules.

\section{CSRBFs}

CSRBFs, firstly introduced by Wendland [20], are used for tuning facial parameters and mapping between facial images within opposite classes. Firstly, Procrustes analysis determines a linear coordinates transformation (translation, reflection, orthogonal rotation, and scaling) of the feature points in the original image to best conform them to the feature points coordinates in the target image. The goodness-of-fit criterion is the sum of squared errors. The Procrustes analysis also returns the minimized value of this dissimilarity measure and the transformed coordinates of the feature points from original image. The coordinates of the transformed images are used to compute the movement of the original feature points and to generate the requested expression by CSRBFs-based image warping.

In general, from given two data sets in $\mathbf{R}^{3}, \boldsymbol{s}_{\mathrm{i}}=\left\{\mathrm{x}_{\mathrm{s}}{ }_{\mathrm{i}}\right.$, $\left.\mathrm{y}_{\mathrm{s}}{ }^{\mathrm{i}}, \mathrm{z}_{\mathrm{s}}{ }_{\mathrm{i}}\right\}$, for a non-deformed object, and $\boldsymbol{d}_{\mathrm{i}}=\left\{\mathrm{x}_{\mathrm{d}}{ }^{\mathrm{i}}, \mathrm{y}_{\mathrm{d}}{ }^{\mathrm{i}}\right.$, $\left.z_{d}^{i}\right\}$, for the deformed object, we construct a space mapping F: $\mathbf{R}^{3} \rightarrow \mathbf{R}^{3}$ which is a CSRBF interpolation of Eq. (1) in each of its components.

$$
F(\bullet)=p(\bullet)+\sum_{i=1}^{N} a_{i} \phi\left(\left\|\bullet-s_{i}\right\|\right)
$$

where, $p$ is a low degree polynomial, $\phi$ is a positive definite and compactly supported radial function in Eq. (2), and $a_{i}$ and $s_{i}$ are the coefficients and centers of the RBFs, respectively. The $r_{0}$ is the support radius.

$$
\varphi(r)=\left\{\begin{array}{cc}
\left(1-r / r_{0}\right)^{2}+ & 0 \leq r \leq r_{0} \\
0 & \text { otherwise }
\end{array}\right.
$$

A SLAE (system of linear algebraic equations) is solved from the displacements of the two data sets to compute RBF coefficients and the coefficients of the low degree polynomial $p$. The warped images are generated from Eq. (1) and reevaluated by two 
subjects in the experimental section.

Fig. 3 shows the face profiles derived from the 63 feature points marked on each portrait and the dissimilarity measure between face profiles computed by Procrustes analysis. The $20 \times 20$ matrix is shown as image and each pixel of the image indicates the minimized value of the dissimilarity measure between the feature points in the firth image and the transformed coordinates of the feature points in the second image. Note that the dissimilarity distance between images is very small. The highest value is shown between pictures 10 and $14(D=0.09125)$. However, evaluations of subjects may not match with these values.

\section{Experimental Results}

For the experimental section, the warped images from the 20 portraits were prepared beforehand. As in the previous work, the participants rated each stimulus on the scale ' 0 '-NO, ' 1 '-DON'T KNOW, ' 2 '-YES to express whether or not they feel the emotion in question. The time for evaluating each stimulus was set to a maximum of $10 \mathrm{~s}$. In each trial, stimulus were selected at random from the set of 20 images and presented for $5 \mathrm{~s}$. The total time for evaluation of each image was also recorded. If the time exceeds $10 \mathrm{~s}$, the image is returned to the set of images in order to be presented one more time. The images evaluated within the maximum time interval of $10 \mathrm{~s}$ are removed from the set. After storing evaluations of each user, the FQHNN was used to learn which are the facial expressions that induce Iyashi on each user. Table 3 shows an example of evaluations made by two subjects.

After FQHNN learning is finished, the system selects which of the rules is closer to user evaluations. Then, it selects one of the images evaluated as 2 and begins to select modified images according to the values of the parameters in the antecedents of the selected rule. For example, in the case of subject 1 , the rule number eight in Table 2, i.e., If ( $x_{6}$ is L) Then Class $($ Iyashi) $=$ YES covered about $60 \%$ of the evaluations in the class. So, the parameter $x_{6}$ was modified to change the evaluation of the subject to the opposite class by using the same image. The image number $5\left(x_{6}=0.0041\right)$ was selected. Fig. 4 shows the set of warped images from image 5 (upper part) and image 6 (lower part). The following sequence of warped images was presented to the subject for evaluation: image $15\left(x_{6}=0.0029\right)$, image $10\left(x_{6}=\right.$ $0.0028)$, and image $8\left(x_{6}=0.0018\right)$. After image 8 was shown the subject evaluated that the image do not produce Iyashi, i.e., Class (Iyashi) $=\mathrm{NO}$ as it was expected.

The rule No.13 in Table 2, i.e., If ( $x_{17}$ is $\left.\mathrm{S}\right)$ Then Class $($ Iyashi) $=$ NO covered more than $50 \%$ of the evaluations in the class. So, the parameter $x_{17}$ was modified to change the evaluation of the subject to the opposite class by using image number $6\left(x_{17}=0.2339\right)$. The following sequence of images was presented to the subject for evaluation: image $3\left(x_{17}=0.2383\right)$, and image $16\left(x_{17}=0.2463\right)$. After image 16 was shown, the subject evaluated that the image produced Iyashi, i.e., Class (Iyashi) = YES as it was expected. In both cases, the starting image is selected randomly. The

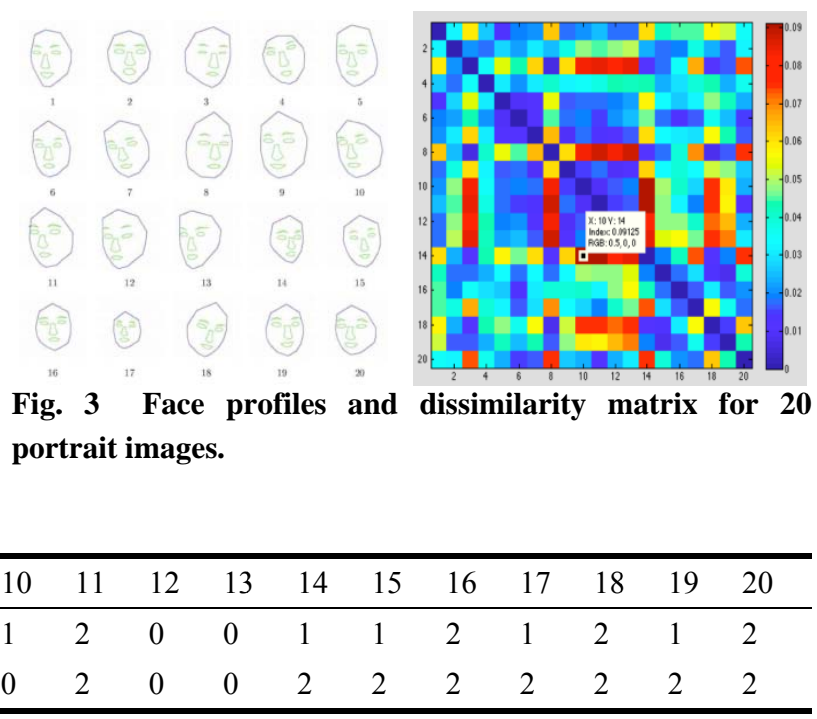

Table 3 Evaluation of 20 portrait images by two subjects.

\begin{tabular}{lllllllllllllllllllll}
\hline & 1 & 2 & 3 & 4 & 5 & 6 & 7 & 8 & 9 & 10 & 11 & 12 & 13 & 14 & 15 & 16 & 17 & 18 & 19 & 20 \\
\hline Subject 1 & 2 & 1 & 0 & 1 & 2 & 1 & 2 & 0 & 2 & 1 & 2 & 0 & 0 & 1 & 1 & 2 & 1 & 2 & 1 & 2 \\
Subject 2 & 2 & 0 & 1 & 0 & 2 & 0 & 2 & 0 & 2 & 0 & 2 & 0 & 0 & 2 & 2 & 2 & 2 & 2 & 2 & 2 \\
\hline
\end{tabular}




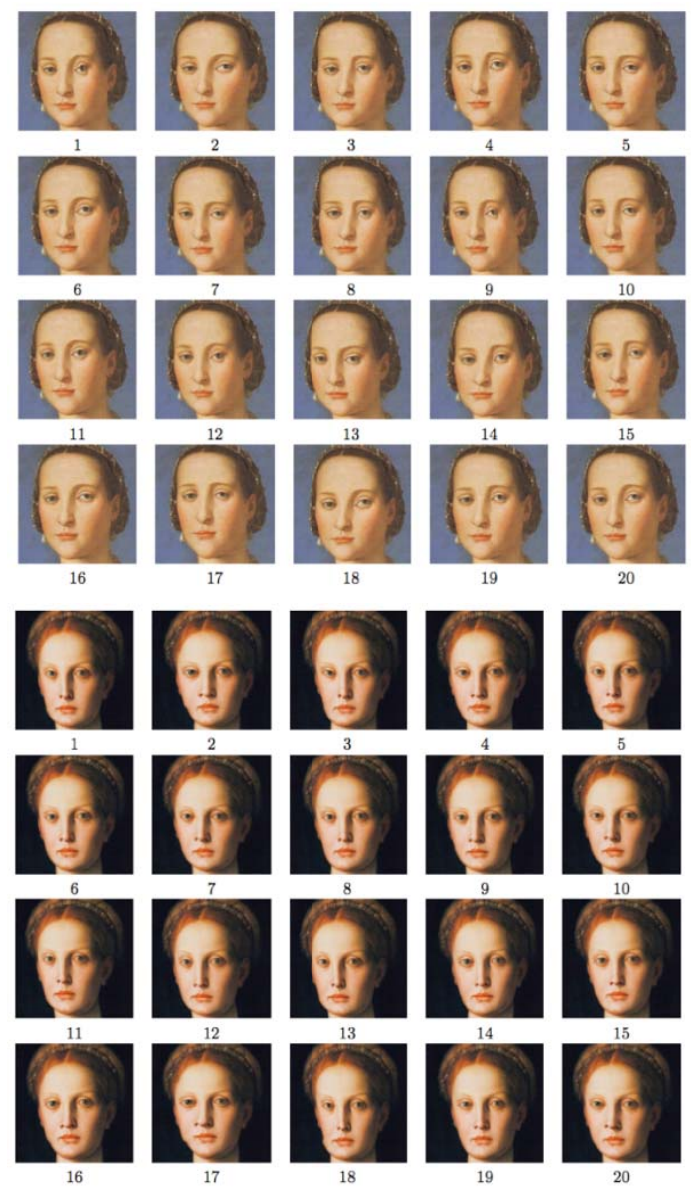

Fig. 4 Warped images used to evaluate the rules generated from the evaluations of subject 1.

experiments have been repeated several times for both subjects using different starting images but similar results are obtained (results not shown).

\section{Conclusions}

This paper is a continuation of our previous works in which a knowledge base of 35 rules was created from the trained FQHNN representing the evaluations of $N=87$ subjects. In order to validate the extracted rules, the parameters influencing the evaluation of $80 \%$ the subjects were selected from the set of rules. Changing only two of the selected parameters (i.e., normalized area of the right eyebrow and the nose) new warped images were generated and evaluated by two subjects. The experimental results showed that although the dissimilarity distance between images is very small, the evaluation of the subjects could be modified to the opposite classification (i.e., Iyashi and Non-Iyashi) by warping original images with CSRBFs.

In this work, we have explored only single parameters influencing subjects evaluations separately. However, we could employ genetic algorithms to explore combinations of parameters and increase the percent of coverage of the rules to explore. An active learning technique to explore the design space is also under development.

\section{Acknowledgment}

Several parts of the research work were carried out by the support of the Grants-in-Aid for scientific research (category C) under Grant No. 26420211. We acknowledge its aid dearly.

\section{References}

[1] Sandhusen, R. L. 2000. "Consumer Behavior, Ser. Chapter 9." Educational Series Inc. 3: 217-43.

[2] Fitzsimons, G., Hutchinson, J., Williams, P., Alba, J., Chartrand, T. L., Huber, J., Kardes, F., Menon, G., Raghubir, P., Russo, J., Shiv, B., and Tavassoli, N. 2002. "Non-conscious Influences on Consumer Choice." Marketing Letters 13 (3): 269-79.

[3] Nagamachi, M. 2011. Kansei/Affective Engineering. Florida: CRC Press.

[4] Bradley, M., and Lang, P. 1994. "Measuring Emotion: The Self-assessment Manikin and the Semantic Differential." J. Behav. Ther. \& Psychiat. 25 (1): 49-59.

[5] Lang, P., Bradley, M., and Cuthbert, B. 2008. International Affective Picture System (IAPS): Affective Ratings of Pictures and Instruction Manual. Technical report A-8, University of Florida, Gainesville, FL.

[6] Mikels, J., Fredrickson, B., Larkin, G. R., Lindberg, C., Maglio, S., and Lorenz, P. R. 2005. "Emotional Category Data on Images from the International Affective Picture System.” Behavior Research Methods 36 (4): 626-30.

[7] Russell, J. A., and Barrett, L. 1999. "Core Affect, Prototypical Emotional Episodes, and Other Things Called Emotion: Dissecting the Elephant." Journal of Personality and Social Psychology 76 (5): 805-19.

[8] Diago, L. A., Kitaoka, T., Hagiwara, I., and Kambayashi, T. 2011. "Neuro-Fuzzy Quantification of Personal Perceptions of Facial Images Based on a Limited Data Set." IEEE Transactions on Neural Networks 22 (12): 2422-34. 
[9] Ekman, P., and Friesen, W. V. 1978. Facial Action Coding System: A Technique for the Measurement of Facial Movement. Palo Alto: Consulting Psychologists Press.

[10] Scherer, K. R. 1992. What Does Facial Expression Express? International Review of Studies on Emotion 2: $139-65$.

[11] Pantic, M., and Bartlett, M. S. 2007. Machine Analysis of Facial Expressions. Viena: I-Tech.

[12] Fasel, B., and Luettin, J. 2003. "Automatic Facial Expression Analysis: A Survey.” Pattern Recognition 36 (1): 259-75.

[13] Tian, Y., Kanade, T., and Cohn, J. 2005. Facial Expression Analysis. New York: Springer.

[14] Kitaoka, T., Diago, L. A., Hagiwara, I., Kitazaki, S., and Yamane, S. 2008. "Definition, Detection and Generation of Iyashi Expressions." Journal of Computational Science and Technology 2 (4): 413-22.

[15] Matsui, T. 2008. "Institutionalization of Consumer Needs: The Case of the "Healing Boom" in Japan." Center for Japanese Business Studies (HJBS),
Hitotsubashi University, Working Paper Series 071.

[16] Benitez, E., Hernandez, A., Reyes, J., Marasinghe, A. 2013. "Kansei Engineering in Gigakuman Character Design." In Proceedings of 2013 International Conference on Biometrics and Kansei Engineering (ICBAKE), 293-6.

[17] Ohira, T., Nakamura, T., Kanoh, M., Kunitachi, T., and Itoh, H. 2009. "A Rough Set Approach to Extract Painting Composition Rules." In Proceedings of IEEE Int. Conference on Fuzzy Systems, 1574 -8.

[18] Ando, M., and Hagiwara, M. 2009. "3D Character Creation System Using Kansei Rule with the Fitness Extraction Method." In Proceedings of IEEE Int. Conference on Fuzzy Systems, 1507-12.

[19] Okuhara, M., Goto, S., Higo, A., and Aoto, T. 2011. "Relationship between Kawaii Feeling and Biological Signals." Transactions of Japan Society of Kansei Engineering 10 (2): 109-14.

[20] Wendland, H. 1995. "Piecewise Polynomial, Positive Defined and Compactly Supported Radial Functions of Minimal Degree." AICM 4: 389-96. 